\title{
The influence of axial compressive stresses on the formability and scattering of process parameters in micro-hydroforming processes of tubes
}

\author{
Christoph Hartla ${ }^{\mathrm{a}}$, Andreas Chlynin, and Max Radetzky \\ Cologne University of Applied Sciences Cologne, Betzdorfer Strasse 2, 50679 Cologne, Germany
}

\begin{abstract}
Feasible product geometries manufactured with micro-hydroforming, as well as process stability are crucially influenced by the microstructure of the used tube material. The higher ratio of grain size to tube wall thickness $d_{k} / t_{0}$ in micro-hydroforming, compared to conventional tube hydroforming, leads to an increase of scattering of process parameters and instabilities. This paper presents experimental and theoretical results for the microhydroforming of tubes made from stainless steel and from platinum with a focus on the correlations between the microstructure of the workpiece material, the stress state during forming and the resulting stability of the forming process. Investigated tube dimensions were $800 \mu \mathrm{m}$ outer diameter and $40 \mu \mathrm{m}$ wall thickness of the steel tubes, respectively $1650 \mu \mathrm{m}$ and $76 \mu \mathrm{m}$ of the platinum tubes. The average ratio $d_{k} / t_{0}$ was about 0.3 for the steel material and about 0.9 for the platinum alloy. It has been shown that superimposed axial stresses are suitable to reduce the restricting influence of an increased ratio $d_{k} / t_{0}$ and to improve the forming result as well as the process stability.
\end{abstract}

\section{Introduction}

Micro-Hydroforming is a down-scaled forming process, based on the expansion of metal tubes with outer diameters below about $1000 \mu \mathrm{m}$ by internal pressurization within a die cavity which corresponds to the final workpiece shape. It was developed to provide an economic technology for the mass production of complex hollow shaped components at micro-scale for the strong demand from numerous industrial sectors on the field of micro-technology. [1, 2]. In particular the medical market is characterized by a use of tubular micro-products which show a great potential to be manufactured by micro-hydroforming.

Similar to other micro-forming processes, so-called size-effects [3] influence the feasible product geometry with micro-hydroforming as well as the process stability. An important cause of size-effects is resulting from the increasing ratio of grain size to tube wall thickness $d_{k} / t_{0}$ in micro-hydroforming when reducing the scale compared to conventional tube hydroforming process size. Experimental bursting tests of micro-tubes and the comparison of the results with tests of macro-tubes in [4] have shown that such influences have to be considered in micro-hydroforming.

The reason for this was provided with the work of [5], where it was shown theoretically by the use of a plain strain crystal plasticity finite element based modelling technique that localized necking of a

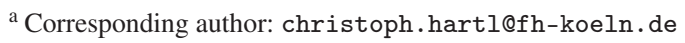

This is an Open Access article distributed under the terms of the Creative Commons Attribution License 4.0, which permits unrestricted use, distribution, and reproduction in any medium, provided the original work is properly cited. 


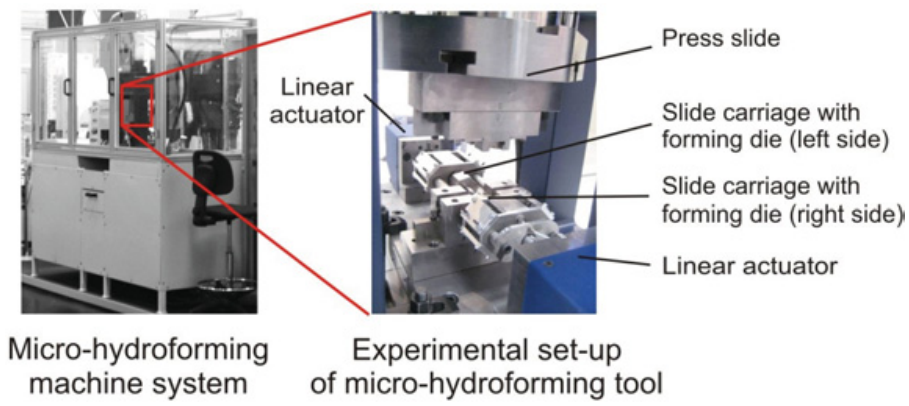

Figure 1. Micro-Hydroforming machine system [1, 8].

micro-tube wall is related to the angle between the crystal slip systems and the hoop stress direction. Consequently, as a result of the small number of grains in tube wall direction, necking and following bursting progresses faster in the expansion of micro-tubes than in macro-tubes which can be regarded as a homogeneous continuum due to their larger number of grains.

For conventional hydroforming it has already been identified that a superimposed axial compressive force improves the formability of the tube metal [6]. This measure reduces the occurrence of instabilities due to necking at an early stage of the process in so far as no other instabilities ensues, caused by an excessive axial force such as wrinkling or buckling of the tube [6]. A first experimental application of axial forces in micro-hydroforming have been already presented by [7] with the objective to investigate suitable sealing systems for the forming of tubes with an outer diameter of $1000 \mu \mathrm{m}$. However, until now no systematic research has been published concerning the influences of superimposed axial forces on the formability for micro-hydroforming processes.

The present paper summarizes experimental results for the micro-hydroforming of tubes made from stainless steel AISI 304 [4] and from the platinum alloy PtIr10 [8], in conjunction with their analysis with the aid of finite element simulations (FEA). Special focus is on the correlations between the microstructure of the workpiece material, the stress state during forming with and without the application of superimposed axial forces and the resulting stability of the forming process.

\section{Experimental set-up}

The micro-hydroforming machine system shown in Fig. 1 was used to conduct the experimental work [1]. It is applicable for the micro-hydroforming of components with cross section dimensions between about $200 \mu \mathrm{m}$ and $2000 \mu \mathrm{m}$ and is equipped with a spindle driven pressure intensifier which enables to apply an internal pressure $p_{i}$ of up to $400 \mathrm{MPa}$. The machine system is provided with three axes for a translatory movement of tool elements: Two linear actuators with spindle gears with a maximum force $F_{a}$ of $800 \mathrm{~N}$, and one hydraulic driven actuator with a maximum force $F_{c}$ of $20 \mathrm{kN}$. The machine is equipped with a flexible control system based on a conventional programmable logic controller system.

The starting tubes used for the experiments were provided in annealed condition with the measured dimensions and mechanical specifications determined by tensile tests of the tubular material as listed in Table 1. To expand the micro-tubes made from AISI 304, the tube ends were clamped without a possible axial movement and pressurized with increasing $p_{i}$, until instability due to necking and subsequent bursting occurred, as described in [4].

The experimental results for the tubes made from PtIr10 are an outcome of a feasibility study, documented in [8], where from a total number of approximately 300 experimental trials those tests were analysed which showed failures due to necking and bursting of the tube wall. Two types of load 
Table 1. Material properties and dimensions of the hydroformed micro-tubes.

\begin{tabular}{|c|c|c|}
\hline & $\begin{array}{c}\text { Stainless steel } \\
\text { tubes }\end{array}$ & $\begin{array}{c}\text { Platinium } \\
\text { tubes }\end{array}$ \\
\hline Material & AISI 304 & PtIr10 \\
\hline Initial yield stress $\sigma_{\mathrm{Y} 0}(\mathrm{MPa})$ & 329 & 250 \\
\hline Tensile strength $\sigma_{\mathrm{UTS}}(\mathrm{MPa})$ & 699 & 346 \\
\hline Tube outer diameter $d_{0}(\mu \mathrm{m})$ & 799 & 1651 \\
\hline Tube wall thickness $t_{0}(\mu \mathrm{m})$ & 39 & 76 \\
\hline Tube length $l_{0}(\mathrm{~mm})$ & 10 & 8 \\
\hline
\end{tabular}

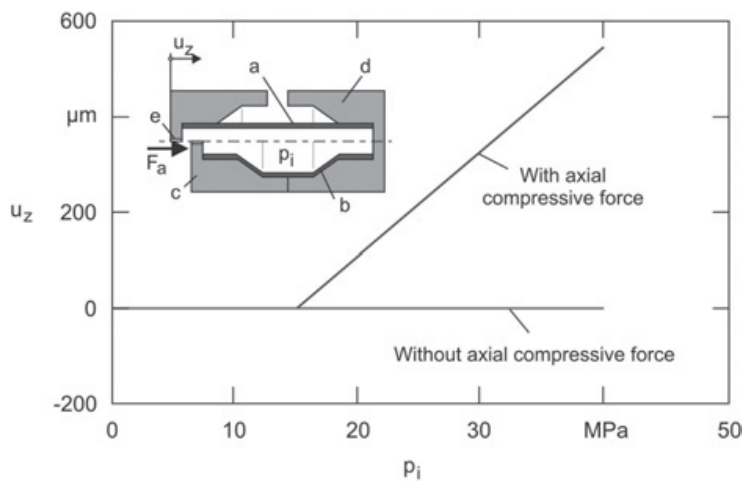

Figure 2. Load paths for platinum tube expansion (a) starting tube, b) hydroformed component, c) left tool half, d) right tool half, e) inlet for pressurizing medium).

paths were applied to these tubes: the first type with only the internal pressure $p_{i}$ as a forming load for the expansion, with the tube ends clamped by the sealing system, and a second with expansion by $p_{i}$ and a superimposed axial compressive force $F_{a}$, caused by an axial stroke $u_{z}$ of the left half of the tools. Figure 2 shows the schematic tool set-up and applied load paths.

\section{Modelling of finite element simulations}

To support the analysis of the experimental results, FE-models of the hydroforming tests were developed, based on a two dimensional, axisymmetric approach. The software ABAQUS with an explicit algorithm was applied to compute the finite element based simulation model which consisted of about 400 elements. 4-node, linear, axisymmetric elements of the type CAX4R were used. The symmetry of the forming process enabled to simplify the model and to calculate merely one halve of the formed component. Furthermore, the part of the tube end located in the tool area where the die cavity dimension corresponds to the outer tube diameter was not included in the model. In this area no notable change in the wall thickness was measured at the experimentally formed components. Hence, material movement and relative movement between the outer surface of the tube end and the tool surface in this area were negligible in the present case. The internal pressure $p_{i}$ was applied as a normal stress to the inner surface of the tube model and the axial stroke $u_{z}$ was implemented by an according displacement of the outer element nodes in the sectional plane of the model as depicted in Fig. 3a. For the simulations without axial compression force, an axial stroke of $u_{z}=0$ was applied.

Homogenous and isotropic elastic-plastic material behaviour with strain hardening was applied in order to establish the ideal forming process without size-effects, according to the material properties 


\section{MATEC Web of Conferences}

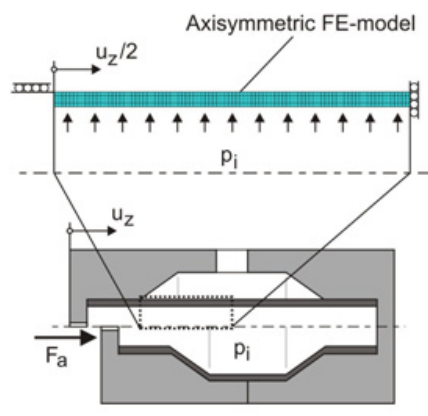

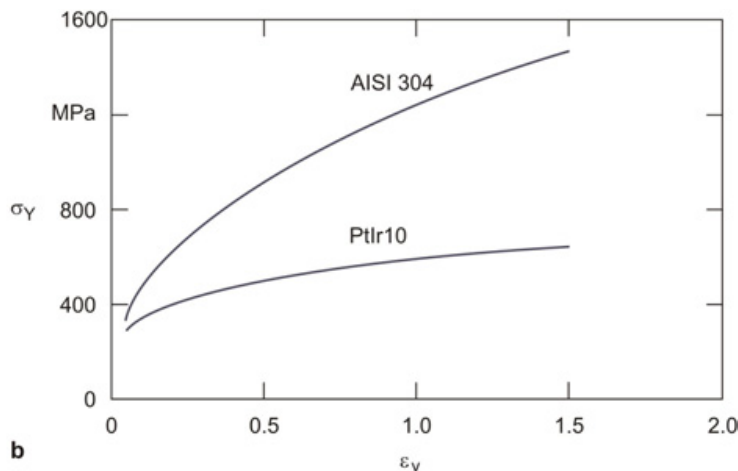

Figure 3. FE-modelling, a) diagram of the model for FE-simulations, b) approximated yield curves of the investigated materials with yield stress $\sigma_{Y}$ versus plastic equivalent strain $\varepsilon_{v}$.

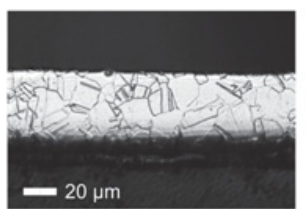

a

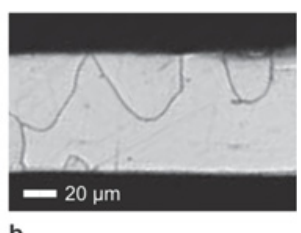

Figure 4. Microstructure of the workpiece materials, a) stainless steel tube (AISI 304), b) platinum tube (PtIr10).

presented in Table 1 and with the yield curves as provided by [9] (Fig. 3b). Upsetting tests of cylindrical specimens were conducted to determine the yield curves.

\section{Results}

The microstructure of the starting tubes was analysed (Fig. 4) and an average grain size $d_{k}$ of $12 \mu \mathrm{m}$ for the stainless steel tube material and $68 \mu \mathrm{m}$ for the platinum tube material was determined. This results in grain size to wall thickness ratios $d_{k} / t_{0}$ of 0.3 and 0.9 respectively. In both cases the grains were equally shaped and randomly orientated without a visible texture. The number of single grains with $d_{k} / t_{0} \approx 1$ was considerably higher for the platinum tubes compared to the workpieces made from stainless steel.

For both types of tubes, the expansion under the applied loads was calculated with the simulation model described before. Figure 5 shows the acting internal pressure $p_{\mathrm{i}}$ versus the radial expansion $r_{x}$ of the tube wall at the maximum point of expansion. Furthermore, the experimentally measured internal pressures for the occurrence of a failure were collected and marked in these diagrams. For the experiments with the stainless steel micro-tubes, measurement data for $r_{\mathrm{x}}$ were derived from the analysed average expansion diameter at bursting [4] and considered in Fig. 5a. The positions of the failure cases along the simulated $p_{\mathrm{i}}-r_{\mathrm{x}}$-curve for the platinum tubes (Fig. $5 \mathrm{~b}$ ) were obtained by projecting the experimentally measured internal pressures at bursting to this curve.

The expansion tests of the stainless steel tubes delivered a scattering of the internal pressure for the occurrence of bursting between 55.7 and $57.4 \mathrm{MPa}$ which corresponds to a standard deviation of $0.55 \mathrm{MPa}$. A considerably higher scattering was determined for the experiments with the platinum tubes. In particular for those, which were formed without superimposed axial force. For these tests, the lowest value of bursting pressure was determined with $11 \mathrm{MPa}$, and the highest with $39 \mathrm{MPa}$. The bursting pressures for the trials with axial compressive force were between 32 and $38.9 \mathrm{MPa}$. The resulting standard deviations are 9.4 MPa and 2.2 MPa respectively. It is evident in Fig. $5 \mathrm{~b}$ that for the forming of 

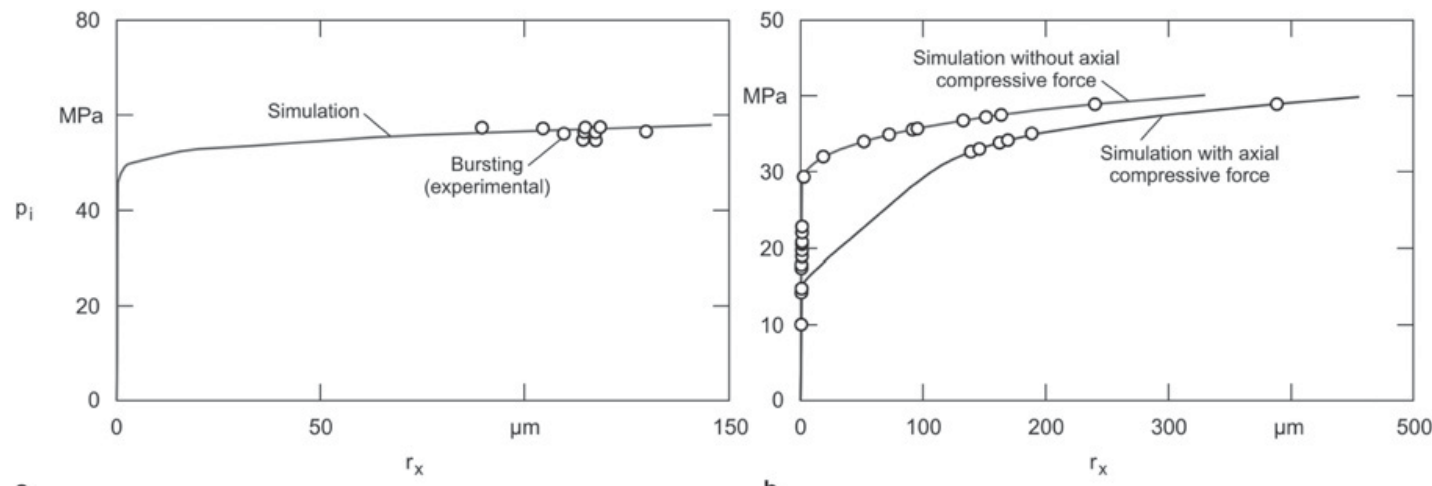

b

Figure 5. Pressure-expansion curves derived from simulations and experimentally determined bursting pressures, a) stainless steel tube (AISI 304), b) platinum tube (PtIr10).

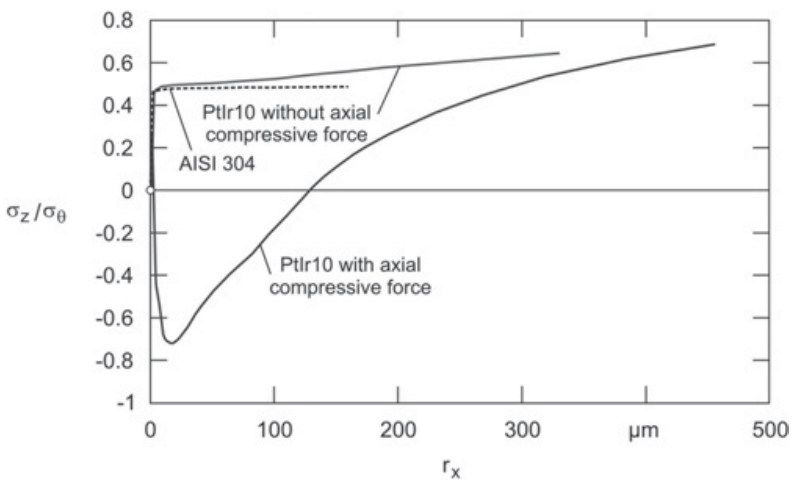

Figure 6. Simulation results of stress-ratio $\sigma_{z} / \sigma_{\theta}$ versus radial expansion $r_{x}$.

the platinum tubes without axial compressive force a considerable number of failures took place before the tubes started to expand. In the cases of the forming of stainless tubes with smaller ratio of grain size to wall thickness and the forming of the platinum tubes with superimposed axial compressive force, failures appeared only at higher internal pressures, when a noticeable expansion took place. The average maximum expansion diameter of the platinum tubes could be increased from about $1800 \mu \mathrm{m}$ without superimposed axial force to about $1970 \mu \mathrm{m}$ with applying an axial force.

It can be assumed that the premature bursting before the theoretical yield start of the expanded tubes made from PtIr10 was caused by the effect described in [5] which results from a comparatively large ratio of material grain size to wall thickness $d_{k} / t_{0}$. In cases where single huge grains are oriented with their crystal slip systems to the hoop stress direction in such a way that the resulting shear stress to move the lattice planes against each other is smaller than the respective shear stress of adjacent grains, local necking and subsequent bursting occur earlier in comparison with homogenous macro-tubes with a larger number of grains. Figure 6 presents the development of the ratio of axial stresses $\sigma_{z}$ to hoop stresses $\sigma_{\theta}$ in the tube wall at the point of maximum expansion during the forming. It can be seen from this figure that the load paths applied to the tubes made from stainless steel and platinum, which do not provide a superimposed axial force, result in a stress ratio $\sigma_{z} / \sigma_{\theta}>0$ along the overall forming process. In contrast to this, a superimposed axial compressive force is resulting in a stress ratio $\sigma_{z} / \sigma_{\theta}<0$ at the 


\section{MATEC Web of Conferences}

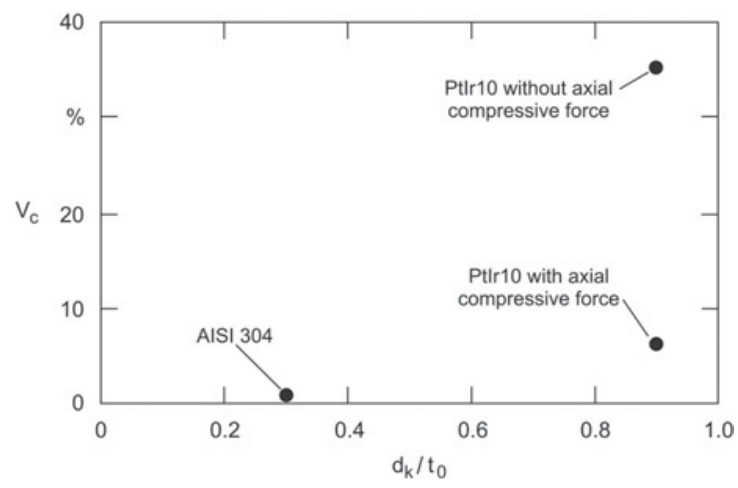

Figure 7. Coefficient of variation $V_{\mathrm{c}}$ versus grain size to wall thickness ratio $d_{\mathrm{k}} / t_{0}$ for the investigated failure cases in micro-tube hydroforming expansions.

first stage of the process, which change at a radial expansion $r_{x}$ of about $130 \mu \mathrm{m}$ to $\sigma_{z} / \sigma_{\theta}>0$ at the last stage (Fig. 6). This is resulting from the fact that at this point the amount of axial tensile stress, caused by the internal pressurization, is exceeding the amount of axial compressive stress, initiated by the superimposed axial compressive force.

The comparison of the development of the simulated stress states in Fig. 6 with the experimental occurrence of failures in Fig. 5 shows that in the case of a superimposed axial compressive force, failures appeared only after the point where the resulting stress ratio $\sigma_{z} / \sigma_{\theta}$ within the tube wall changed from negative to positive values. The fact that size-effects due to the large ratio of grain size to tube wall thickness increase the danger of premature necking and bursting, and that none of these failures was determined for the investigated samples within the area of $\sigma_{z} / \sigma_{\theta}<0$, leads to the recommendation that a superimposed axial compressive force should be applied in micro-hydroforming of a maximum feasible amount within the limits of wrinkling and buckling of the tube wall. Figure 7 summarizes the results by comparing the coefficient of variation resulting from the standard deviation and average value of the determined internal pressures at occurrence of failures. It is obvious from this figure that small ratios of grain size to tube wall thickness and the application of superimposed axial forces contribute to a higher process stability and reduced scattering.

\section{Conclusions}

In this work, investigations about the the occurrence of failures caused by localised necking and following bursting in micro-hydroforming of tubes made from stainless steel and platinum is presented. Load paths for the hydroforming of the specimens were applied taking into account both forming with and without a superimposed axial compression force. In both cases, the measured values of the internal pressures in the event of instabilities showed a distinct scattering, with however a reduced deviation between the individual test results for the hydroforming with a superimposed axial force. Furthermore, the application of an axial compression force avoided the premature bursting of the formed tubes below the theoretical yield limit, which was detected for a considerable number of the expansion tests without axial force. Additionally, the feasible expansion diameter was increased. With the aid of finite element simulations it was found that, within the frame of the investigated process parameters and number of specimens, no failures occurred as long as axial compressive stresses were acting within the formed tube wall. An analysis of the microstructure of the tube material used showed an increasing scattering with increasing ratio of grain size to tube wall thickness. It was concluded that the scattering of the measured 
internal pressure at the occurrence of failures can be attributed to the size-effects resulting from the amount of this ratio.

\section{References}

[1] C. Hartl, G. Anyasodor, Steel Research Int. 81, 9 (2010)

[2] Y. Qin, A. Brockett, Y. Ma, J. Zhao, C. Harrison, W. Pan, X. Dai, D. Loziak, Int. J. Adv. Manuf. Technol. 47 (2010)

[3] F. Vollertsen, D. Biermann, H.H. Hansen, I.S. Jawahir, K. Kuzman, CIRP Annals 58, 2 (2009)

[4] C. Hartl, G. Anyasodor, J. Lungershausen, in Proc. of Int. ESAFORM Conf. Metal Forming 2011, Belfast (2011)

[5] W. Zhuang, S. Wang, J. Lin, D. Balint, C. Hartl, Europ. J. Mechanics A/Solids 31 (2011)

[6] F. Dohmann, in Umformtechnik, edited by K. Lange, vol. 4 (Springer, Berlin, 1993)

[7] G. Ngaile, J. Lowrie,J. Micro Nano-Manuf. 2, 4 (2014)

[8] C. Hartl, H. Schiefer, A. Chlynin, Manufacturing Rev. 1, 17 (2014)

[9] N.N., VDI-Standard 3200, Issue 3 (The Association of German Engineers, Beuth, Berlin, 1982) 